\title{
Metastatic Thymoma of the Breast
}

\author{
Sung Mok Kim, MD \\ Eun Young Ko, MD' \\ Boo-Kyung Han, MD' \\ Jung Hee Shin, MD' \\ Seok Seon Kang, MD ${ }^{1}$ \\ Seok-Jin Nam, MD \\ Eun Yoon Cho, $\mathrm{MD}^{3}$
}

Breast metastasis from nonmammary malignant neoplasms is uncommon, and it accounts for approximately $2 \%$ of all breast tumors. Distant metastasis of thymoma is very rare, and especially to extrathorcic areas. We report a female who had a metastatic thymoma in her breast 20 years after undergoing resection for a non-invasive thymoma. She presented with a palpable mass in her left breast. Mammography and ultrasonogram showed a lobular mass at the anterior glandular portion. Histological examination after surgical excision revealed a metastatic thymoma.

\section{Index terms :}

Breast neoplasm

Breast metastasis

Metastatic thymoma

Recurrent thymoma

DOI:10.3348/kjr.2008.9.1.80

Korean J Radiol 2008; 9:80-83

Received June 22, 2006; accepted after revision January 23, 2007.

'Department of Radiology and the Center for Imaging Science, ${ }^{2}$ Department of Surgery, and ${ }^{3}$ Department of Pathology, Samsung Medical Center Sungkyunkwan University School of Medicine, 50 llwon-dong, Kangnam-ku, Seoul 135-710, Korea

Address reprint requests to: Eun Young Ko, MD, Department of Radiology and Center for Imaging Science, Samsung Medical Center, Sungkyunkwan University School of Medicine, 50 llwon-dong, Kangnam-gu, Seoul 135-710, Korea.

Tel. (822) 3410-6418

Fax. (822) 3410-0084

e-mail: claudel@skku.edu
7 he breast is the most common site of primary malignant tumors in adult women and it is unusual site for nonmammary malignancies (1). The incidence of breast metastasis from other organs ranges from $1.7 \%$ to $6.6 \%$ in autopsy series and is even lower $(1.2-2.7 \%)$ in clinical and cytological series $(2,3)$. Yet the surgical planning and follow-up change depending on if a tumor is a primary or metastatic breast tumor, and it is the important to recognize the clinical and imaging differences between them.

Thymoma is usually a slow-growing tumor. Although intrathoracic recurrence sometimes occurs in patients with invasive thymoma, few cases of distant metastasis following resection of a non-invasive thymoma have been reported (4), and metastatic thymic tumor to the breast is extremely rare indeed (5).

We describe here the radiological and histological findings of metastatic thymoma of the breast.

\section{CASE REPORT}

A 49-year-old woman visited our hospital for a palpable mass in her left breast. Physical examination revealed a 2-cm firm mass in the upper outer quadrant of the left breast. There was no palpable axillary lymphadenopathy.

She had a history of operation 20 years ago for mediastinal non-invasive thymoma without metastasis. She developed cough and blood tinged sputum two years previously. Chest X-ray and CT scan showed a mass in the right upper lung. After one year, with the worsening symptoms and increasing size of mass, she underwent lobectomy for the right upper lung lesion and this was diagnosed as metastatic thymoma, type B3 with pleural invasion. She also received radiation therapy after lobectomy. Six months after radiation therapy, a palpable mass developed in her left breast. The patient did not experience any symptoms of myasthenia gravis.

Mammography showed a high-density mass, $2.3 \mathrm{~cm}$, with irregular shaped in the left upper outer breast (Fig. 1). On ultrasound (US), a hypoechoic mass, $2.6 \mathrm{~cm}$, with 


\section{Breast Metastasis from Thymoma}

irregular shaped was noted in the junction of the breast parenchyma and the subcutaneous fat layer (Fig. 2A).

Color Doppler US showed increased vascularity within the mass (Fig. 2B). US-guided core needle biopsy and surgical excision showed a metastatic thymoma of the B3 type. On microscopic examination, the tumor was predominantly composed of solid nests of oval or polygonal epithelial cells

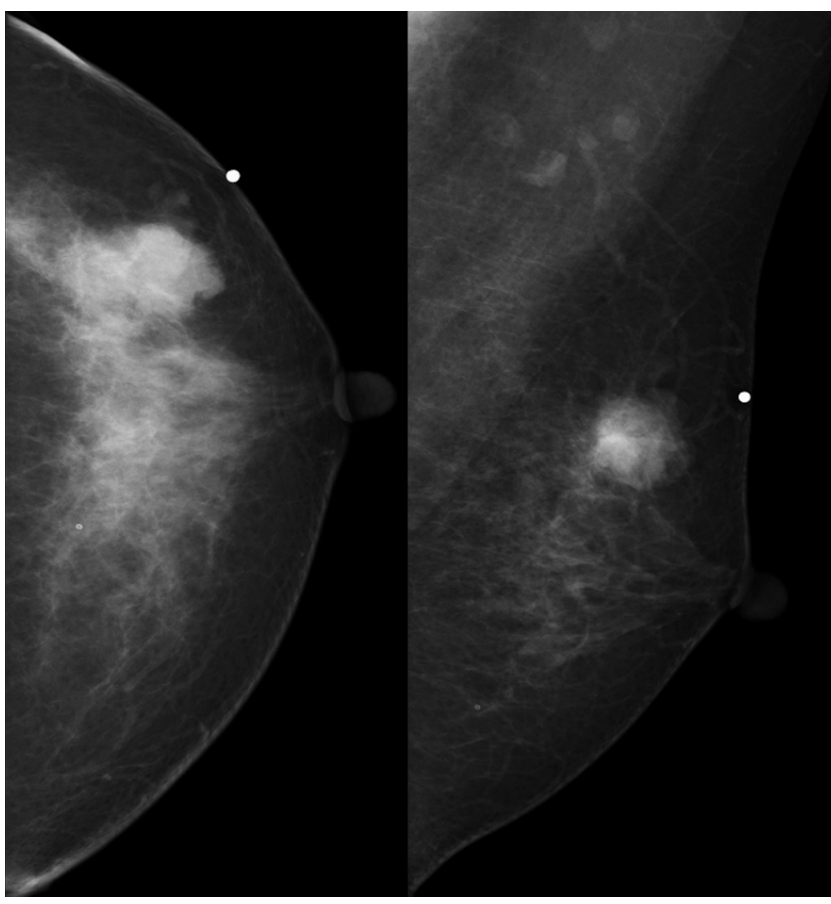

Fig. 1. A 49-year-old female patient with a palpable mass in her left breast. Mammography demonstrates a high-density mass with irregular shaped, measuring $2.3 \mathrm{~cm}$, without calcification in the upper outer quadrant of the left breast. separated by dense fibrous septa. The nests were admixed with a minor component of lymphocytes, resulting in a lobular sheet-like growth of the neoplastic epithelial cells (Fig. 3A). On immunohistochemistry staining, the tumor cells were positive for CK (AE1/AE3) and CD99, and TdTpositive thymocytes were found in the tumor cell nests (Fig. 3B).

After that, she underwent additional excision for a newly developed tiny nodule in the left breast, which was also a metastatic nodule. Additional chemotherapy or radiation therapy was not considered.

\section{DISCUSSION}

Metastatic disease to the breast from nonmammary primary lesions is rather unusual. The breast had resistance to metastasis from extramammary malignancy because it contained large areas of fibrous tissue with a relatively poor blood supply (6); however, virtually any malignancy can metastasize to the breast. The most frequent source of such metastasis was reported to be melanoma, but a wide variety of other tumors may secondarily involve the breast, including leukemia, lymphoma, carcinomas from ovary, lung, esophagus, stomach, colon, liver, cervix and the nasopharynx, uterine leiomyosarcoma and embryonal rhabdomyosarcoma $(1,2)$.

Reports about metastatic breast tumors from the thymus are extremely rare and there is one report about poorly differentiated thymic carcinoma with invasion and distant metastasis to the breast that was found within six months during the period of adjuvant chemotherapy (5). Our case

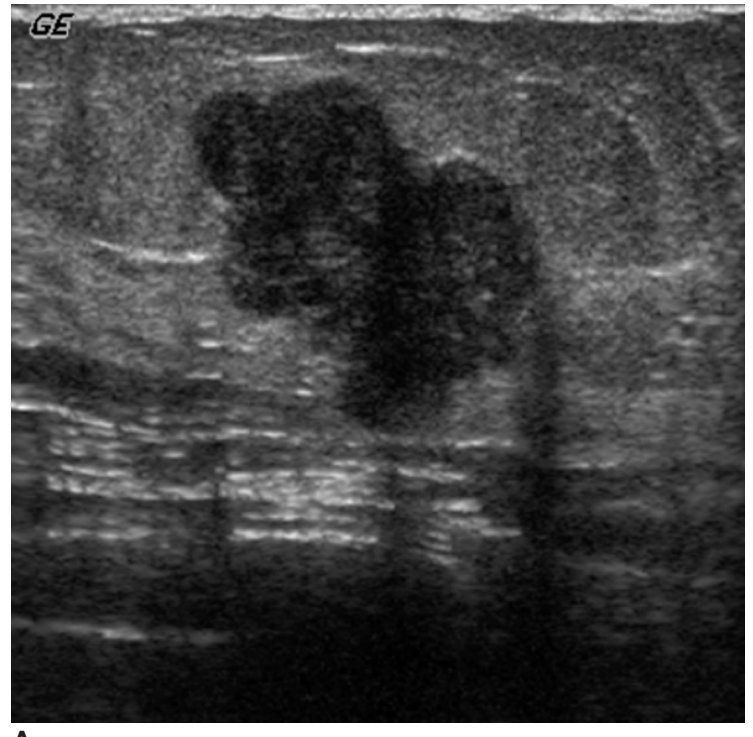

A

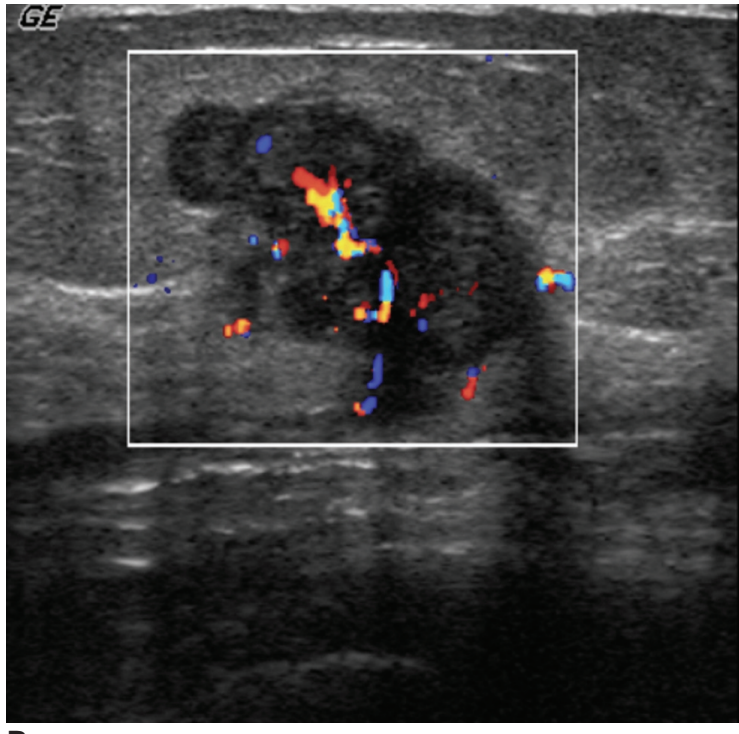

B

Fig. 2. Ultrasound (A) of her left breast shows a hypoechoic mass, 2.6-cm, with irregular shaped, located in the junction of the breast parenchyma and the subcutaneous fat layer. Color Doppler ultrasound (B) shows increased vascularity within the mass. 

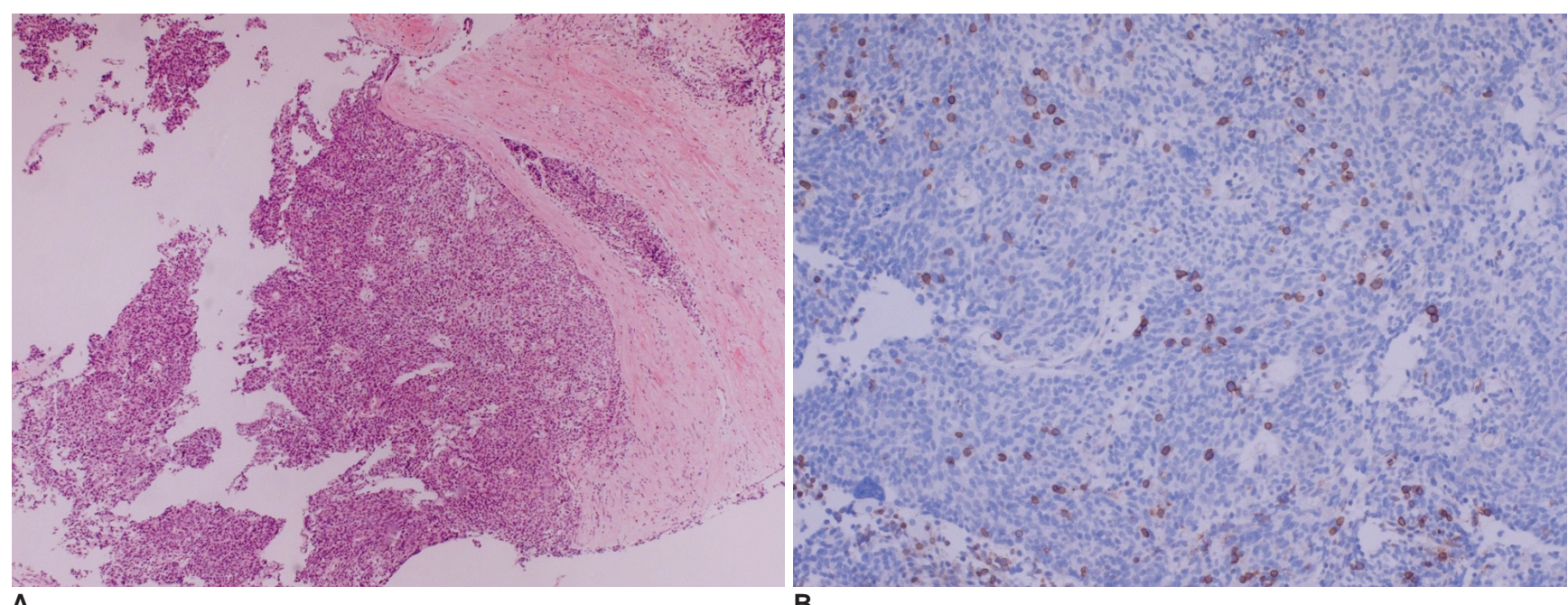

A

Fig. 3. Photomicrograph $(\mathbf{A})$ discloses lobular growth of the oval to polygonal cells that are separated by dense fibrous stroma (Hematoxylin \& Eosin staining, $\times 100)$. Immunohistochemical staining (B) demonstrates the presence of thymocytes in the tumor cell nests $($ CD99, $\times 100)$.

initially showed non-invasive thymoma without metastasis, and the women experienced more than 18 years of a disease-free period until detection of the distant metastasis.

Metastatic lesions to breast can be single or multiple, unilateral or bilateral. They are usually round or oval masses with well-circumscribed to ill-defined margins on mammography (7). The US findings are round, hypoechoic lesions in a superficial location without acoustic shadowing or microcalcification (1).

Our case was a well-defined, lobular hypoechoic solid mass and it was located at the junction of the breast parenchyma and the subcutaneous fat tissue. There was no spiculation, thick hyperechoic halo, nor parenchymal distortion around the mass that would suggest primary malignancy of the breast. As compared with the usual metastatic masses, our case showed a more lobulating contour and heterogeneous internal echo texture on US, and this may reflect the growth pattern of the primary tumor. Histological type B thymoma grows in expansile sheets or it more often displays a lobular architecture that recapitulates the normal thymic cortex (8). However, the radiological findings of extrathoracic solid organ metastasis from a thymic epithelial tumor are not known, and the one previously reported case showed slightly different features from ours.

Thymic epithelial tumor is a distinctive pathologic entity that exhibits variable histologic features and heterogeneous oncologic behavior. Recently, a new classification of the World Health Organization that's based on the morphology of the epithelial cells as well as on the lymphocyte-to-epithelial cell ratio has been adopted because of the good correlation between the histologic appearance and the oncologic behavior (9). According to the WHO classification, our case is a B3 thymoma and it is a high-risk thymoma with a $30 \%$ incidence of invasive tumor (10).

Metastases from thymoma usually occur within the thorax and only $3 \%$ of patients have extrathoracic lesions (11). One study reported that the mean interval between detecting primary tumor and detecting extrathoracic metastasis was nine years, and the maximum interval was 21 years (11). This suggests that thymic tumor can metastasize even a long time after treatment of the initial mediastinal mass, along with it growing very slowly. Therefore, when the patient with a breast mass has a history of thymic tumor, metastasis from thymic tumor should be included in a differential diagnosis regardless of the initial low malignant potential of thymic tumor or the long diseasefree period.

\section{References}

1. Yeh $\mathrm{CN}$, Lin $\mathrm{CH}$, Chen MF. Clinical and ultrasonographic characteristics of breast metastases from extramammary malignancies. Am J Surg 2004;70:287-290

2. Toombs BD, Kalisher L. Metastatic disease to the breast: clinical, pathologic, and radiographic features. AJR Am J Roentgenol 1977;129:673-676

3. Amichetti M, Perani B, Boi S. Metastases to the breast from extramammary malignancies. Oncology 1990;47:257-260

4. Nomori H, Watanabe K, Ohtsuka T, Naruke T, Suemasu K, Orikasa $\mathrm{H}$, et al. Pulmonary metastasis 12 years after resection of thymoma with microscopic capsule invasion. Jpn J Clin Oncol 2004;34:630-633

5. Youk JH, Kim EK, Kim MJ, Oh KK, Park YN. Metastatic breast lesion from thymic carcinoma. J Ultrasound Med 2006;25:13391342

6. Jochimsen PR, Brown RC. Metastatic melanoma in the breast 


\section{Breast Metastasis from Thymoma}

masquerading as fibroadenoma. JAMA 1976;236:2779-2780

7. Cardenosa G. Breast Imaging. Lippincott: Williams \& Wilkins, 2004

8. World Health Organization Classification of Tumours, Pathology \& Genetics Tumours of the Lung, Pleuera, Thymus and Heart. Travis WD, Brambilla E, Muller-Hermelink HK, and Harris CC. Lyon, France, International Agency for Research on Cancer, 2004

9. Han J, Lee KS, Yi CA, Kim TS, Shim YM, Kim J, et al. Thymic epithelial tumors classified according to a newly established
WHO scheme: CT and MR findings. Korean J Radiol 2003;4:4653

10. Sadohara J, Fujimoto K, Miiller NL, Kato S, Takamori S, Ohkuma K, et al. Thymic epithelial tumors: comparison of CT and MR imaging findings of low-risk thymomas, high-risk thymomas, and thymic carcinomas. Eur J Radiol 2006;60:70-79 11. Lewis JE, Wick MR, Scheithauer BW, Bernatz PE, Taylor WF. Thymoma. A clinicopathologic review. Cancer 1987;60:27272743 